\title{
Beryllium abundances in stars hosting giant planets ${ }^{\star}$
}

\author{
N. C. Santos ${ }^{1}$, R. J. García López ${ }^{2,3}$, G. Israelian², M. Mayor ${ }^{1}$, R. Rebolo ${ }^{2,4}$, A. García-Gil ${ }^{2}$, \\ M. R. Pérez de Taoro ${ }^{2}$, and S. Randich ${ }^{5}$ \\ 1 Observatoire de Genève, 51 Ch. des Maillettes, 1290 Sauverny, Switzerland \\ 2 Instituto de Astrofísica de Canarias, 38200 La Laguna, Tenerife, Spain \\ 3 Departamento de Astrofísica, Universidad de La Laguna, Av. Astrofísico Francisco Sánchez s/n, \\ 38206 La Laguna, Tenerife, Spain \\ 4 Consejo Superior de Investigaciones Científicas, Spain \\ 5 INAF/Osservatorio Astrofisico di Arcetri, Largo Fermi 5, 50125 Firenze, Italy
}

Received 26 October 2001 / Accepted 22 February 2002

\begin{abstract}
We have derived beryllium abundances in a wide sample of stars hosting planets, with spectral types in the range $\mathrm{F} 7 \mathrm{~V}-\mathrm{K} 0 \mathrm{~V}$, aimed at studying in detail the effects of the presence of planets on the structure and evolution of the associated stars. Predictions from current models are compared with the derived abundances and suggestions are provided to explain the observed inconsistencies. We show that while still not clear, the results suggest that theoretical models may have to be revised for stars with $T_{\text {eff }}<5500 \mathrm{~K}$. On the other hand, a comparison between planet host and non-planet host stars shows no clear difference between both populations. Although preliminary, this result favors a "primordial" origin for the metallicity "excess" observed for the planetary host stars. Under this assumption, i.e. that there would be no differences between stars with and without giant planets, the light element depletion pattern of our sample of stars may also be used to further investigate and constraint $\mathrm{Li}$ and Be depletion mechanisms.
\end{abstract}

Key words. stars: abundances - stars: chemically peculiar - stars: evolution - planetary systems

\section{Introduction}

In the last few years we have witnessed a fantastic development in an "old" but until recently not very successful field of astrophysics: the search for extra-solar planets. Following the first success in exoplanet searches with the discovery of the planet around $51 \mathrm{Peg}$ (Mayor \& Queloz 1995), the number of known giant planets orbiting solartype stars did not stop growing, being currently of 72 (including 7 multi-planetary systems $)^{1}$.

Unexpectedly, the planets found to date do not have much in common with the ones in our own Solar System (for a review see Marcy et al. 2000; Udry et al. 2001;

Send offprint requests to: N. C. Santos,

e-mail: Nuno.Santos@obs. unige.ch

* Based on observations collected with the VLT/UT2 Kueyen telescope (Paranal Observatory, ESO, Chile) using the UVES spectrograph (Observing runs 66.C-0116 A and 66.D$0284 \mathrm{~A}$ ), and with the William Herschel and Nordic Optical Telescopes, operated at the island of La Palma by the Isaac Newton Group and jointly by Denmark, Finland, Iceland, and Norway, respectively, in the Spanish Observatorio del Roque de los Muchachos of the Instituto de Astrofísica de Canarias.

1 Counts of January 2002. For a list see e.g. table at obswww. unige.ch/ naef/who_discovered_that_planet.html or Mayor \& Santos 2001). One remarkable characteristic appears to be related with the parent stars themselves: stars with planetary companions are considerably metalrich when compared with single field dwarfs (Gonzalez 1998; Santos et al. 2000; Gonzalez et al. 2001; Santos et al. 2001a, 2001b). To explain the observed difference two main explanations have been suggested. The first and more "traditional" is based upon the fact that the more metals you have in the proto-planetary disk, the higher should be the probability of forming a planet (see e.g. Pollack et al. 1996 for the traditional paradigm of planetary formation). Thus, in this case the "excess" of metallicity is seen as primordial to the cloud that gave origin to the star/planet system. "Opposing" to this view, the high metal content observed for stars with planets has also been interpreted as a sign of the accretion of high- $Z$ material by the star sometime after it reached the main-sequence (e.g. Gonzalez 1998; Laughlin 1996).

Although recent results seem to favor the former scenario as the key process leading to the observed metal richness of stars with planets (Santos et al. 2001a, 2001b; Pinsonneault et al. 2001), signs of accretion of planetary material have also been found for some planet hosts (e.g. Israelian et al. 2001a; Laws \& Gonzalez 2001). 


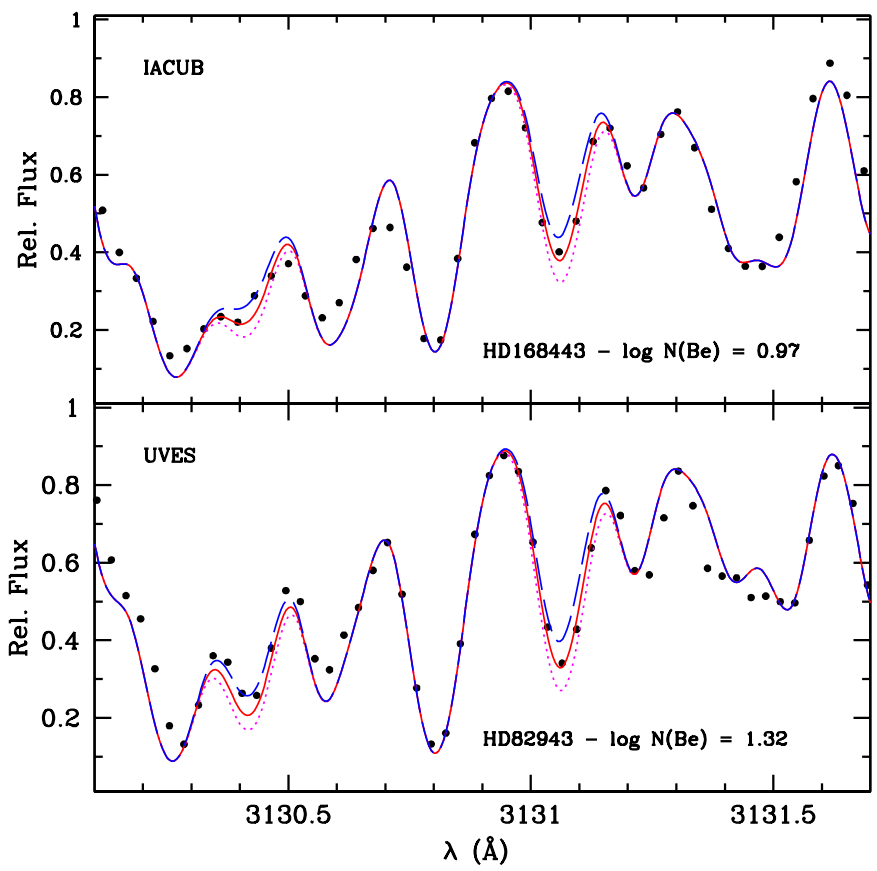

Fig. 1. Spectra in the BeII line region (dots) for two of the objects observed, and three spectral synthesis with different Be abundances, corresponding to the best fit (solid line) and to changes of \pm 0.2 dex, respectively.

The question is then turned to know how frequent those phenomena happen, and to how much these could have affected the observed metal contents.

One possible and interesting approach to this problem may pass by the study of one particularly important element: beryllium (Be). Together with lithium $(\mathrm{Li})$ and boron $(\mathrm{B}), \mathrm{Be}$ is a very important tracer of the internal stellar structure and kinematics. Be is mainly produced by spallation reactions in the interstellar medium, while it is burned in the hot stellar furnaces (e.g. Reeves 1994). While most works of light element abundances are based on $\mathrm{Li}$ (the abundances of this element are easier to measure), Be studies have one major advantage when compared with Li. Since it is burned at much higher temperatures, Be is depleted at lower rates than $\mathrm{Li}$, and thus we can expect to measure Be abundances in stars which have no detectable $\mathrm{Li}$ in their atmospheres (like intermediateage late $\mathrm{G}$ - or K-type dwarfs). In fact, for about $50 \%$ of the known planet host stars no Li was detected (Israelian et al. 2001b). Furthermore, Li studies have shown the presence of a significant scatter for late-type stars of similar temperature. This has also been observed in open clusters where all stars have the same age, and appears to be related to the clusters's age, rotational velocities, pre-Main Sequence history, etc. (see, e.g., García López et al. 1994; Randich et al. 1998; Jones et al. 1999), a fact that may complicate or even preclude a comparison.

Given all these points, Be studies of planetary host stars can indeed be particularly important and telling. For example, if pollution has played some important role in determining the high-metal content of planet host stars,

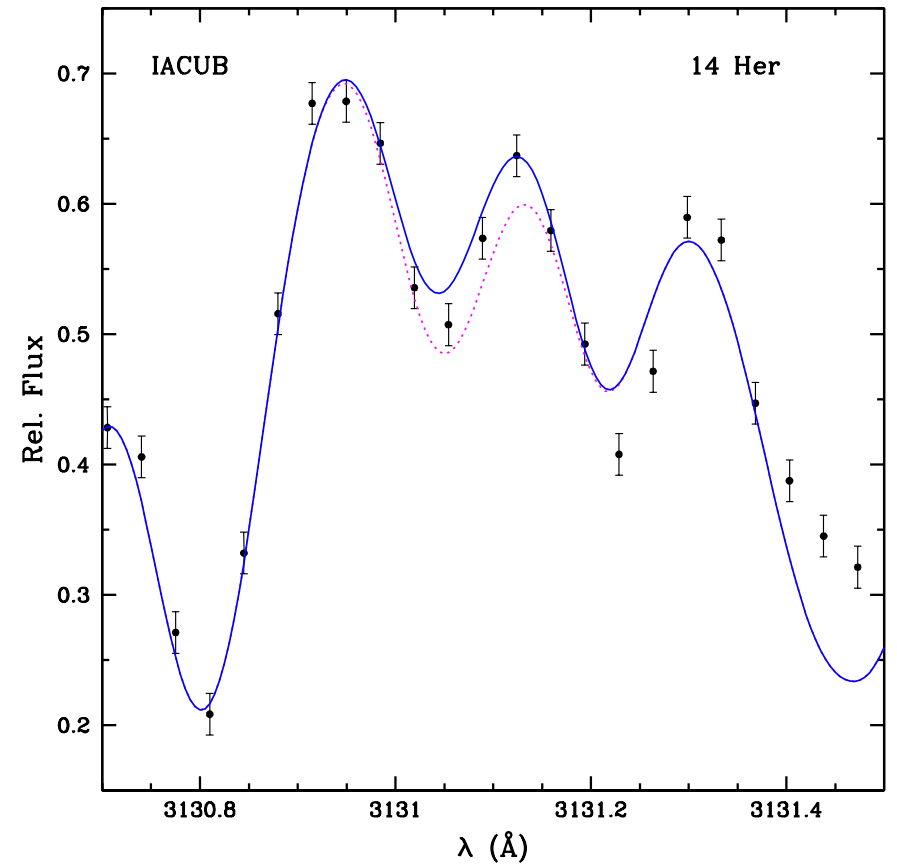

Fig. 2. Spectrum in the Be II line region (dots) for $14 \mathrm{Her}$ (HD 145675), and two spectral synthesis with different Be abundances, corresponding to no Be (solid line) and to $\log N(\mathrm{Be})=0.5$. Error-bars represent the photon noise error. A conservative upper limit of 0.5 for the Be abundance was considered.

we would expect to find a similar or even higher increase in the Be contents. This is basically due to the fact that planetary material is relatively poor in $\mathrm{H}$ and $\mathrm{He}$ when compared to the star (e.g. Anders \& Grevesse 1989). Furthermore, and unlike for iron, Be may be already a bit depleted in the stellar surface. Thus, the injection of planetary material into this latter could even be responsible for a more important abundance change in the Be abundance than in the iron content. If pollution has indeed played an important role, the net result of the fall of planetary material into the central "sun" would thus be that, for a given temperature interval, we should find that planet hosts are (in principle) more Be-rich that non-planet host stars. In other words, the analysis of Be abundances represents an independent way of testing the pollution scenario.

García López \& Péres de Taoro (1998) carried out the first Be measurements in stars hosting planets: 16 Cyg A and B, and 55 Cnc, followed by Deliyannis et al. (2000). In order to continue to address this problem, we present ${ }^{2}$ here a study of Be abundances in a set of 29 stars with planets, and a smaller set of 6 stars without known planetary companions. In Sects. 2 and 3 we present the observations and analysis of the data and in Sect. 4 we discuss the results in the context of the planetary host stars chemical abundances, but also in terms of the Be depletion processes. We conclude in Sect. 5 .

${ }^{2}$ Preliminary results were already presented in García López et al. (2001). 


\section{Observations and data reduction}

Observations of the BeII doublet at $3131 \AA$ were carried out during 5 different observing runs. Two of them made use of the UVES spectrograph, at the VLT/UT2 Kueyen telescope (ESO, Chile). The obtained spectra have a resolution $R \equiv \lambda / \Delta \lambda$ of around 70000 for one of the runs (66.C-0116 A) and 50000 for the other (66.D$0284 \mathrm{~A}$ ), and the $S / N$ ratios in the region around $3131 \AA$ varying between 30 and 250 (from now on we will refer to runs $\operatorname{UVES}(\mathrm{A}) /(\mathrm{B})$, respectively). UVES spectra were complemented with data obtained using the UES spectrograph $(R=55000)$ at the 4.2-m William Herschel Telescope (WHT) and, in two different observing runs (A/B), with the IACUB echelle spectrograph $(R=33000)$ at the 2.6-m Nordic Optical Telescope (NOT), both at the Observatorio del Roque de los Muchachos (La Palma). The observations, together with the corresponding observing run and $S / N$ ratios obtained are listed in Table 1.

Data reduction was done using $\mathrm{IRAF}^{3}$ tools in the echelle package. Standard background correction, flatfield, and extraction procedures were used. For the UVES(A) run and for the UES data, the wavelength calibration was done using a ThAr lamp spectrum taken during the same night. For the UVES(B) and IACUB runs the wavelength calibration was done using photospheric lines in the region of interest. The final linear dispersions for the UVES(A), UVES(B), IACUB(A), IACUB(B), and UES spectra were $30,17,35,35$ and $17 \mathrm{~mA}$ pixel $^{-1}$, respectively, with rms values in the range $4 \times 10^{-4}$ to $8 \times 10^{-3} \AA$.

\section{Abundance analysis}

\subsection{Atmospheric parameters}

García López et al. (1995) carried out a detailed analysis of the sources of uncertainties regarding the determination of Be abundances. They concluded that the precision of the derived Be abundances is mostly dependent on the choice of the stellar atmospheric parameters. In particular, they are very sensitive to the adopted value for the surface gravity $(\log g)$.

In order to limit the possible systematic errors in our determinations it is important, whenever possible, to use an uniform set of atmospheric parameters for all the programme stars. We thus decided to use the values listed by Santos et al. (2001a, 2001b), computed from an uniform and accurate spectroscopic analysis available for most of the stars studied in this paper ${ }^{4}$. For three of the stars (HD 870, HD 1461, and HD 3823 for which no planetary

\footnotetext{
${ }^{3}$ IRAF is distributed by National Optical Astronomy Observatories, operated by the Association of Universities for Research in Astronomy, Inc., under contract with the National Science Foundation, USA.

${ }^{4}$ The list of atmospheric parameters in Santos et al. (2001a) was determined by these authors for part of the objects, or was taken from the studies of Gonzalez (1998), Gonzalez \& Laws (1998), and Gonzalez et al. (1999, 2001).
}

companions were found to date), no parameters were available, and we computed them using CORALIE or FEROS spectra, in the very same way as in Santos et al. (2001a). The values are listed in Table 2 . As discussed by the authors, the errors in $T_{\text {eff }}$ are usually lower than $50 \mathrm{~K}$, and errors in the microturbulence parameter are of the order of $0.1 \mathrm{~km} \mathrm{~s}^{-1}$. As for $\log g$, the uncertainties are in the range of 0.10 to $0.15 \mathrm{dex}^{5}$.

\subsection{Spectral synthesis}

The abundance analysis was done in standard Local Thermodynamic Equilibrium (LTE) using a revised version of the code MOOG (Sneden 1973), and a grid of Kurucz et al. (1993) ATLAS9 atmospheres. Be abundances were derived by fitting synthetic spectra to the data, using the same line-list as in García López \& Perez de Taoro (1998). While both Be II lines at 3130.420 and $3131.065 \AA$ are present in our data, we only used the latter, given the severe line blending in the region around $3130.420 \AA$ (used only for checking the consistency of the fit).

In the analysis, the overall metallicity was scaled to the iron abundance. We then iterated by changing the Be abundance, the continuum placement and the Gaussian smoothing profile until the best fit for the whole spectral region was obtained (we fitted all the spectrum between 3129.5 and $3132.0 \AA$ ). When considered important (e.g. for $\tau$ Boo), the smoothing function used was a combination of a Gaussian and a rotational profile; for these cases we used the $v \sin i$ value determined from the width of the CORAVEL cross-correlation dip (Benz \& Mayor 1984). Three examples are shown in Figs. 1 and 2. The resulting abundances for all the objects observed are listed in Table 2. Here we use the notation $\log N(\mathrm{Be})=[\mathrm{Be}]=\log (\mathrm{Be} / \mathrm{H})+12$.

\subsection{Errors}

It is not simple to derive accurate uncertainties for measurements of Be abundances (García López et al. 1995). In this paper the errors were estimated as follows. We considered that from the errors of $\pm 50 \mathrm{~K}$ in temperature and \pm 0.15 dex in $\log g$ we can expect typical uncertainties around 0.03 and 0.06 dex, respectively. There are several OH lines blended with the Be II $3130.420 \AA$ line; changes in the oxygen abundance would also change the location of the pseudo-continuum in that region, affecting the overall fit. To take this into account, an error of $0.05 \mathrm{dex}$, associated with the uncertainties in the oxygen abundances expected for these stars, has been added. Other atmospheric parameters, like the metallicity $[\mathrm{Fe} / \mathrm{H}]$ and the microturbulence, do not influence significantly the results, and we will conservatively consider that together they introduce an error of 0.05. Adding quadratically, these figures

\footnotetext{
${ }^{5}$ Note that these small uncertainties are relative, and not absolute.
} 
Table 1. Observing log for stars with and without planets.

\begin{tabular}{|c|c|c|c|c|c|}
\hline $\begin{array}{l}\text { HD } \\
\text { number }\end{array}$ & Star & $\bar{V}$ & $\begin{array}{l}\text { Observ. } \\
\text { Run }\end{array}$ & $\overline{S S / N}$ & Date \\
\hline \multicolumn{6}{|c|}{ Stars with planets: } \\
\hline HD 13445 & Gl 86 & 6.1 & UVES(A) & 150 & Nov. 2000 \\
\hline HD 16141 & HD 16141 & 6.8 & $\operatorname{UVES}(\mathrm{A})$ & 120 & Nov. 2000 \\
\hline HD 17051 & $\iota$ Hor & 5.4 & $\operatorname{UVES}(\mathrm{A})$ & 150 & Nov. 2000 \\
\hline HD 52265 & HD 52265 & 6.3 & UVES(A) & 120 & Dec. 2000 \\
\hline HD 75289 & HD 75289 & 6.4 & $\operatorname{UVES}(\mathrm{A})$ & 110 & Dec. 2000 \\
\hline HD 82943 & HD 82943 & 6.5 & $\operatorname{UVES}(\mathrm{A})$ & 140 & Jan. 2001 \\
\hline HD 210277 & HD 210277 & 6.5 & $\operatorname{UVES}(\mathrm{A})$ & 110 & Nov. 2000 \\
\hline HD 217107 & HD 217107 & 6.1 & $\operatorname{UVES}(\mathrm{A})$ & 120 & Nov. 2000 \\
\hline- & $\mathrm{BD}-103166$ & 10.0 & UVES(B) & 20 & Feb. 2001 \\
\hline HD 38529 & HD 38529 & 5.9 & UVES(B) & 60 & Feb. 2001 \\
\hline HD 75289 & HD 75289 & 6.4 & UVES(B) & 30 & Feb. 2001 \\
\hline HD 92788 & HD 92788 & 7.3 & UVES(B) & 40 & Feb. 2001 \\
\hline HD 82943 & HD 82943 & 6.5 & UVES(B) & 35 & Feb. 2001 \\
\hline HD 108147 & HD 108147 & 7.0 & UVES(B) & 60 & Feb. 2001 \\
\hline HD 121504 & HD 121504 & 7.5 & UVES(B) & 45 & Feb. 2001 \\
\hline HD 134987 & HD 134987 & 6.5 & UVES(B) & 60 & Feb. 2001 \\
\hline HD 95128 & $47 \mathrm{UMa}$ & 5.1 & $\operatorname{IACUB}(\mathrm{A})$ & 100 & May 2000 \\
\hline HD 114762 & HD 114762 & 7.3 & $\operatorname{IACUB}(\mathrm{A})$ & 65 & May 2000 \\
\hline HD 117176 & $70 \mathrm{Vir}$ & 5.0 & $\operatorname{IACUB}(\mathrm{A})$ & 70 & May 2000 \\
\hline HD 130322 & HD 130322 & 8.0 & $\operatorname{IACUB}(\mathrm{A})$ & 35 & May 2000 \\
\hline HD 145675 & $14 \mathrm{Her}$ & 6.7 & $\operatorname{IACUB}(\mathrm{A})$ & 65 & May 2000 \\
\hline HD 168443 & HD 168443 & 6.9 & $\operatorname{IACUB}(\mathrm{A})$ & 55 & May 2000 \\
\hline HD 187123 & HD 187123 & 7.9 & $\operatorname{IACUB}(\mathrm{A})$ & 55 & May 2000 \\
\hline HD 195019 & HD 195019 & 6.9 & $\operatorname{IACUB}(\mathrm{A})$ & 50 & May 2000 \\
\hline HD 10697 & 109 Psc & 6.3 & $\operatorname{IACUB}(B)$ & 40 & Oct. 2001 \\
\hline HD 12661 & HD 12661 & 7.4 & $\operatorname{IACUB}(\mathrm{B})$ & 40 & Oct. 2001 \\
\hline HD 22049 & $\epsilon$ Eri & 3.7 & $\operatorname{IACUB}(B)$ & 100 & Oct. 2001 \\
\hline HD 9826 & $v$ And & 4.1 & UES & 120 & Aug. 1998 \\
\hline HD 120136 & $\tau$ Boo & 4.5 & UES & 90 & Aug. 1998 \\
\hline HD 143761 & $\rho \mathrm{CrB}$ & 5.4 & UES & 120 & Aug. 1998 \\
\hline HD 217014 & $51 \mathrm{Peg}$ & 5.5 & UES & 100 & Aug. 1998 \\
\hline \multicolumn{6}{|c|}{ Stars without known planets: } \\
\hline HD 870 & HD 870 & 7.2 & $\operatorname{UVES}(\mathrm{A})$ & 130 & Nov. 2000 \\
\hline HD 1461 & HD 1461 & 6.5 & $\operatorname{UVES}(\mathrm{A})$ & 120 & Nov. 2000 \\
\hline HD 1581 & HD 1581 & 4.2 & $\operatorname{UVES}(\mathrm{A})$ & 140 & Dec. 2000 \\
\hline HD 3823 & HD 3823 & 5.9 & UVES(A) & 130 & Oct. 2000 \\
\hline HD $26965 \mathrm{~A}$ & $o^{2}$ Eri & 4.4 & $\operatorname{IACUB}(B)$ & 55 & Oct. 2001 \\
\hline HD 222335 & HD 222335 & 7.2 & UVES(A) & 110 & Dec. 2000 \\
\hline
\end{tabular}

produce an uncertainty of $0.09 \mathrm{dex}$, that was added to the error due to continuum placement and fit quality for each case, that we conservatively considered to be at least of 0.10 dex. The final errors, listed in Table 2 together with the derived Be abundances, are of the order of 0.16 dex, and quite independent of the $S / N$ of the spectrum.

Note that we are interested in carrying out a differential analysis, and thus the knowledge of the absolute temperatures and surface gravities is not very important. Rather, it is crucial that these values are all in the same "scale".

\section{Be in stars with planets}

There are many evidences indicating that the depletion of $\mathrm{Be}$ is connected with the rotational history of a star (e.g. Stephens et al. 1997). Although still not completely established, this history may be related to the presence or not of a (massive) proto-planetary disk (Edwards et al. 1993; Strom 1994; Stassun et al. 1999; Barnes et al. 2001; Rebull 2001; Hartmann 2002). If true, this may result in different depletion rates for stars which had different disk masses, and thus may or not have been able to form 
Table 2. Stellar atmospheric parameters and resulting beryllium abundances for each spectrum.

\begin{tabular}{|c|c|c|c|c|c|c|}
\hline Star & $T_{\text {eff }}$ & $\log g$ & {$[\mathrm{Fe} / \mathrm{H}]$} & $\log N(\mathrm{Be})$ & Run & $\log N(\mathrm{Li}) \dagger$ \\
\hline \multicolumn{7}{|c|}{ Stars with planets $\dagger \uparrow$ : } \\
\hline $\mathrm{BD}-103166$ & 6320 & 4.38 & 0.33 & $<0.55$ & UVES(B) & - \\
\hline HD 9826 & 6140 & 4.12 & 0.12 & $0.99 \pm 0.18$ & UES & 2.26 \\
\hline HD 10697 & 5605 & 3.96 & 0.16 & $1.38 \pm 0.18$ & $\operatorname{IACUB}(B)$ & 1.94 \\
\hline HD 12661 & 5715 & 4.45 & 0.35 & $1.07 \pm 0.14$ & $\operatorname{IACUB}(\mathrm{B})$ & $<0.99$ \\
\hline HD 13445 & 5205 & 4.70 & -0.20 & $<0.52$ & $\operatorname{UVES}(\mathrm{A})$ & $<0.5$ \\
\hline HD 16141 & 5805 & 4.28 & 0.15 & $1.27 \pm 0.14$ & UVES(A) & $<0.73$ \\
\hline HD 17051 & 6225 & 4.65 & 0.25 & $1.02 \pm 0.17$ & UVES(A) & 2.63 \\
\hline HD 22049 & 5135 & 4.70 & -0.07 & $0.70 \pm 0.20$ & IACUB(B) & $<0.3$ \\
\hline HD 38529 & 5675 & 4.01 & 0.39 & $<0.30$ & UVES(B) & $<0.61$ \\
\hline HD 52265 & 6100 & 4.29 & 0.24 & $1.21 \pm 0.14$ & UVES(A) & 2.73 \\
\hline HD 75289 & 6135 & 4.43 & 0.27 & $1.39 \pm 0.14$ & UVES(A) & 2.84 \\
\hline HD 75289 & 6135 & 4.43 & 0.27 & $1.41 \pm 0.16$ & UVES(B) & 2.84 \\
\hline HD 75289 (avg) & & & & 1.40 & & \\
\hline HD 82943 & 6025 & 4.54 & 0.33 & $1.32 \pm 0.14$ & UVES(A) & 2.52 \\
\hline HD 82943 & 6025 & 4.54 & 0.33 & $1.22 \pm 0.14$ & UVES(B) & 2.52 \\
\hline HD 82943 (avg) & & & & 1.27 & & \\
\hline HD 92788 & 5775 & 4.45 & 0.31 & $1.13 \pm 0.16$ & UVES(B) & - \\
\hline HD 95128 & 5800 & 4.25 & 0.01 & $1.13 \pm 0.14$ & $\operatorname{IACUB}(\mathrm{A})$ & 1.71 \\
\hline HD 108147 & 6265 & 4.59 & 0.20 & $1.02 \pm 0.14$ & $\operatorname{UVES}(\mathrm{B})$ & 2.34 \\
\hline HD 114762 & 5950 & 4.45 & -0.60 & $0.97 \pm 0.14$ & $\operatorname{IACUB}(\mathrm{A})$ & 2.26 \\
\hline HD 117176 & 5500 & 3.90 & -0.03 & $0.82 \pm 0.14$ & $\operatorname{IACUB}(\mathrm{A})$ & 1.76 \\
\hline HD $120136 \dagger \uparrow \dagger$ & 6420 & 4.18 & 0.32 & $0.14 \pm 0.30$ & UES & $<1.07$ \\
\hline HD 121504 & 6090 & 4.73 & 0.17 & $1.40 \pm 0.16$ & UVES(B) & 2.66 \\
\hline HD 130322 & 5410 & 4.47 & 0.05 & $1.07 \pm 0.20$ & $\operatorname{IACUB}(\mathrm{A})$ & $<0.57$ \\
\hline HD 134987 & 5715 & 4.33 & 0.32 & $1.12 \pm 0.17$ & $\operatorname{UVES}(\mathrm{B})$ & $<0.69$ \\
\hline HD 143761 & 5750 & 4.10 & -0.29 & $0.95 \pm 0.19$ & UES & 1.30 \\
\hline HD 145675 & 5300 & 4.27 & 0.50 & $<0.5$ & $\operatorname{IACUB}(\mathrm{A})$ & $<0.7$ \\
\hline HD 168443 & 5555 & 4.10 & 0.10 & $0.97 \pm 0.15$ & $\operatorname{IACUB}(\mathrm{A})$ & $<0.71$ \\
\hline HD 187123 & 5830 & 4.40 & 0.16 & $0.98 \pm 0.14$ & $\operatorname{IACUB}(\mathrm{A})$ & 1.20 \\
\hline HD 195019 & 5830 & 4.34 & 0.09 & $1.27 \pm 0.15$ & $\operatorname{IACUB}(\mathrm{A})$ & $<1.05$ \\
\hline HD 210277 & 5575 & 4.44 & 0.23 & $1.05 \pm 0.17$ & $\operatorname{UVES}(\mathrm{A})$ & $<0.73$ \\
\hline HD 217014 & 5795 & 4.41 & 0.21 & $0.98 \pm 0.14$ & UES & 1.30 \\
\hline HD 217107 & 5660 & 4.42 & 0.39 & $1.01 \pm 0.17$ & $\operatorname{UVES}(\mathrm{A})$ & $<0.86$ \\
\hline \multicolumn{7}{|c|}{ Stars from García López \& Perez de Taoro (1998): } \\
\hline HD 75732 A & 5150 & 4.15 & 0.29 & $<0.55$ & - & $<0.04$ \\
\hline HD 186408 & 5750 & 4.20 & 0.11 & $1.10 \pm 0.17$ & - & 1.24 \\
\hline HD 186427 & 5700 & 4.35 & 0.06 & $1.30 \pm 0.17$ & - & $<0.46$ \\
\hline \multicolumn{7}{|c|}{ Stars without known planets: } \\
\hline HD 870 & 5425 & 4.59 & -0.03 & $0.84 \pm 0.16$ & UVES(A) & $<0.35$ \\
\hline HD 1461 & 5785 & 4.47 & 0.18 & $1.20 \pm 0.17$ & UVES(A) & $<0.71$ \\
\hline HD 1581 & 5940 & 4.44 & -0.15 & $1.19 \pm 0.14$ & UVES(A) & 2.35 \\
\hline HD 3823 & 5950 & 4.12 & -0.27 & $0.98 \pm 0.14$ & UVES(A) & 2.44 \\
\hline HD 26965A & 5185 & 4.73 & -0.26 & $0.56 \pm 0.20$ & IACUB(B) & $<0.22$ \\
\hline HD 222335 & 5310 & 4.64 & -0.10 & $0.87 \pm 0.18$ & UVES(A) & $<0.35$ \\
\hline
\end{tabular}

$\dagger$ The values for the $\mathrm{Li}$ abundances were taken from Israelian et al. (2001b); we refer to this work for references. These authors used the very same set of stellar parameters as in this paper, and thus the listed Li and Be abundances are based on the same parameter scale.

$\dagger \dagger$ We refer to obswww.unige.ch/ naef/who_discovered_that_planet.html for the planet discovery references. $\dagger \dagger \dagger$ For this star ( $\tau$ Boo) the uncertainty in $\log N(\mathrm{Be})$ is higher due to the "large" $v \sin i \sim 15 \mathrm{~m} \mathrm{~s}^{-1}$.

the now discovered giant planets. This could, in fact, have been the case for the pair of very similar dwarfs 16 Cyg A and B (the latter having a detected planetary companion), for which the Li abundances seem to be quite different (Cochran et al. 1997; King et al. 1997; Gonzalez et al. 1998), but show similar amounts of Be 
(García López \& Perez de Taoro 1998). On the other hand, if pollution plays any role, we might also expect to detect some differences between stars with planets and stars without planets concerning light element abundances, and in particular concerning Be.

Stars with planets might thus present statistically different Be contents when compared with stars without (detected) planetary systems.

In Fig. 3 we show a plot of Be abundances for the stars presented in this paper as a function of $T_{\text {eff }}$. Filled symbols represent stars with planets, while open symbols denote dwarfs with no planet companions found by radial-velocity surveys (i.e. measured in the context of the CORALIE survey (Udry et al. 2000), and having no clear radial-velocity signature of a planet). We also included three objects from the study of García López \& Perez de Taoro (1998) - triangles, namely 16 Cyg A (HD 186408), 16 Cyg B (HD 186427) and 55 Cnc (HD $75732 \mathrm{~A}$ ), for which the atmospheric parameters have been computed using the same technique as for the stars presented here (Gonzalez 1998). In order to keep this work as an homogeneous comparative study, we have preferred not to introduce measurements for stars "without" planets taken from other works (e.g. Stephens et al. 1997), derived using different sets of atmospheric parameters and chemical analysis (e.g. equivalent widths).

In the figure, stars with a surface gravity $\log g \leq$ 4.1 (probably slightly evolved) are represented by the squares. These include HD 10697, HD 38529, HD 117176 , HD 143761, and HD 168443 (all planet hosts). Note also that HD 114762, a quite metal-poor dwarf $([\mathrm{Fe} / \mathrm{H}]=-0.6)$ with a brown-dwarf companion was also included amongst the planet hosts.

Superposed with the measurements are a set of Yale beryllium depletion isochrones from Pinsonneault et al. (1990) for solar metallicity and an age of 1.7 Gyr. Given that it is not possible to know what was the initial Be abundance for each star, we have considered an initial $\log N(\mathrm{Be})=1.28$, i.e., between Solar $(\log N(\mathrm{Be})=1.15-$ Chmielewski et al. 1975) and meteoritic $(\log N(\mathrm{Be})=1.42$ - Anders \& Grevesse 1989).

Although still preliminary, a look at the figure shows that the current results argue against pollution as the key process leading to the metallicity excess of stars with planets (see also Santos et al. 2001a, 2001b; Pinsonneault et al. 2001). Adding 50 earth masses of $\mathrm{C} 1$ chondrites to the Sun, for example, would increase its iron abundance by about $0.25 \mathrm{dex}$ (a value similar to the average difference observed between stars with and without detected giant planets), and its Be abundance would increase by a slightly higher factor ${ }^{6}$. No such difference seems present in our data. In the same way, the results also do not support extra-mixing due to an eventual different angular

${ }^{6}$ For this calculation we have used a $\mathrm{Be} / \mathrm{Fe}$ ratio similar to $\mathrm{C} 1$ chondrites, and considered an iron content of $\sim 20 \%$ by mass (Anders \& Grevesse 1989). Note also that the fall of a few jupiter-mass jovian planet(s) would not have a big effect on the iron or beryllium abundance of the star.

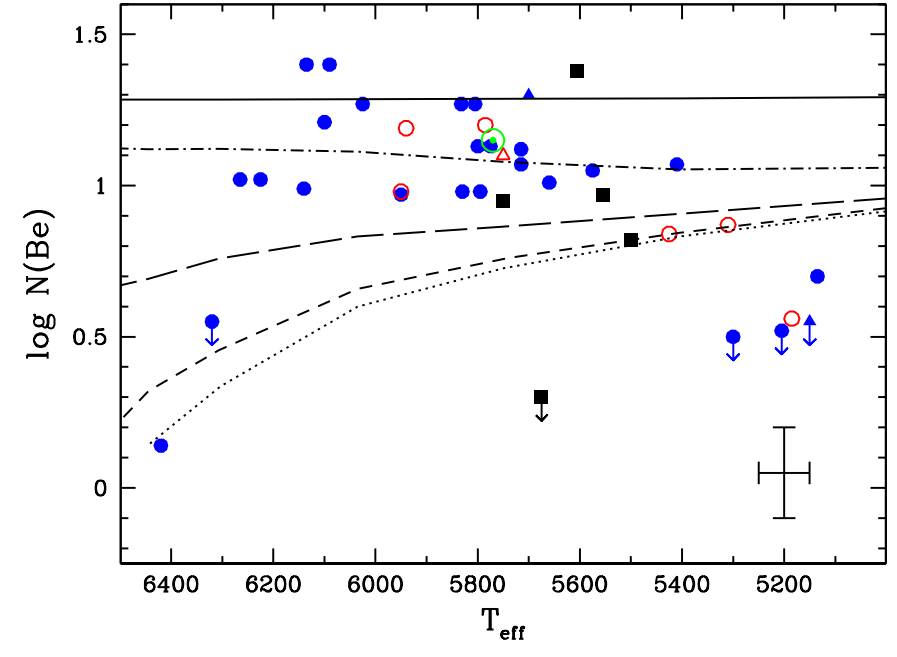

Fig. 3. Be abundances for stars with planets (filled symbols) and stars without detected planetary companions (open symbols) as a function of $T_{\text {eff }}$. Circles represent the stars studied in this work, while triangles denote the three objects taken from the study of García López \& Perez de Taoro (1998). Stars with surface gravities $\log g \leq 4.1$ are denoted by squares. Superposed are the Be depletion isochrones (case A) of Pinsonneault et al. (1990) for solar metallicity and an age of 1.7 Gyr. The Sun is represented by the Solar Symbol. From top to bottom, the lines represent a standard model (solid line), and 4 models with different initial angular momentum (Tables 3 to 6 ). Typical error bars are represented in the lowerright corner of the figure.

momentum history of the two "populations" of stars. We note, however, that at this stage we are limited by the small number of comparison stars analyzed in this work.

In this context, one particular star (HD 82943) deserves a few comments. This planet host was recently found to have a near meteoritic ${ }^{6} \mathrm{Li} /{ }^{7} \mathrm{Li}$ ratio (Israelian et al. 2001a), better interpreted as a sign that planet/planetary material was engulfed by this dwarf sometime during its lifetime. Israelian et al. have suggested that the $\mathrm{Li}$ isotopic ratio of HD 82943 could be explained by the fall of either the equivalent of a 2 jupiter mass giant planet or a 3 earth mass terrestrial planet (or planetary material). Considering that HD 82943 had initially a solar Be abundance, the addition of this quantity of material would increase its Be abundance by about 0.1 dex. A small "excess" of Be would thus be observed, but this value would be within the error bars of our measurements. As we can see from Fig. 3, the Be abundance of HD 82943 is not particularly high when compared to other stars in the plot (although it seems to occupy a position near the upper envelope of the Be abundances). No further conclusions can be taken.

\subsection{The $\log N(B e)$ vs. $T_{\text {eff }}$ trend}

According to the standard models (without rotation - e.g. Table 1 of Pinsonneault et al. 1990), basically no Be depletion should occur for dwarfs in the temperature region 
plotted in the figure. This is clearly not compatible with the observations. However, it is already known that standard models are not able to explain most of the observed behaviors of $\mathrm{Li}$ and $\mathrm{Be}$ (e.g. Stephens et al. 1997). On the other hand, models with rotation (Pinsonneault et al. $1990)^{7}$ were shown to be quite satisfactory for stars with $6500 \mathrm{~K} \leq T_{\text {eff }} \leq 5600 \mathrm{~K}$ (Stephens et al. 1997). But as we can see from the figure, they do not predict significant burning at temperatures around $5200 \mathrm{~K}$. We note that according to these isochrones, Be depletion is done at moderate rates once the star reaches the main-sequence (about 0.06 dex from 0.7 to $1.7 \mathrm{Gyr}$ for a $0.9 M_{\odot}$ dwarf, and at a considerably lower rate for higher ages following the Fig. 11 of Stephens et al. 1997), and thus we do not expect a crucial difference with the presented curves.

In contrast with the models ${ }^{8}$, one very interesting detail is seen. If, for temperatures between about 5600 and $6200 \mathrm{~K}$, the dispersion in the $\log N(\mathrm{Be})$ is remarkably small, and the abundances seem to be consistent with the model predictions, the general trend for lower temperatures follows a slow decrease with decreasing temperature. Under the assumption that no difference exists between stars with and without giant planets, either the trend is due to some metallicity or age effect, or it is simply telling us that the models are not able to reproduce the observations for temperatures bellow $\sim 5600 \mathrm{~K}$. This problem was also noted by Stephens et al. (1997), but no such extreme cases had been found, maybe because their "solar metallicity sample" did not go down to temperatures lower than $\sim 5500 \mathrm{~K}$. Note that the iron abundances for the stars in the plot are in the range from -0.6 to +0.5 , and the variation of the initial Be abundance with stellar metallicity seems to be quite small in this interval (Rebolo et al. 1988; Molaro et al. 1997; García López et al., in preparation).

Particularly interesting are the 4 cases for which only upper values for the Be abundances were found: HD 38529 , HD 145675 (14 Her), HD 13445 (Gl 86), and HD 75732 $(55 \mathrm{Cnc})$, all in the temperature interval between $5150 \mathrm{~K}$ and $5700 \mathrm{~K}$. A detail of the spectral synthesis for one of these stars can be seen in Fig. 2. While for HD 38529 the Be depletion may be explained by the fact that this star is already leaving the main-sequence (its low surface gravity and its position in the HR-diagramme - e.g. Gonzalez et al. 2001 - show it to be evolved) for the three remaining objects no simple explanations seem to exist.

One possible explanation for Be abundances of the most discrepant objects could be their ages. For example, $55 \mathrm{Cnc}$ and $\mathrm{Gl} 86$ seem to be quite old, with isochrone ages in agreement with the oldest stars in the galaxy (ages were determined from theoretical isochrones of Schaller et al. 1992, and Schaerer et al. 1993a, 1993b). This is not the

\footnotetext{
7 Improved Yale models (e.g. Deliyannis \& Pinsonneault 1993) do not show an important difference. The 1990 models were thus used in the current paper, since they are the only tabulated.

${ }^{8}$ We will assume for now that the initial Be abundance was similar (within the uncertainties) for all the stars in the plot.
}
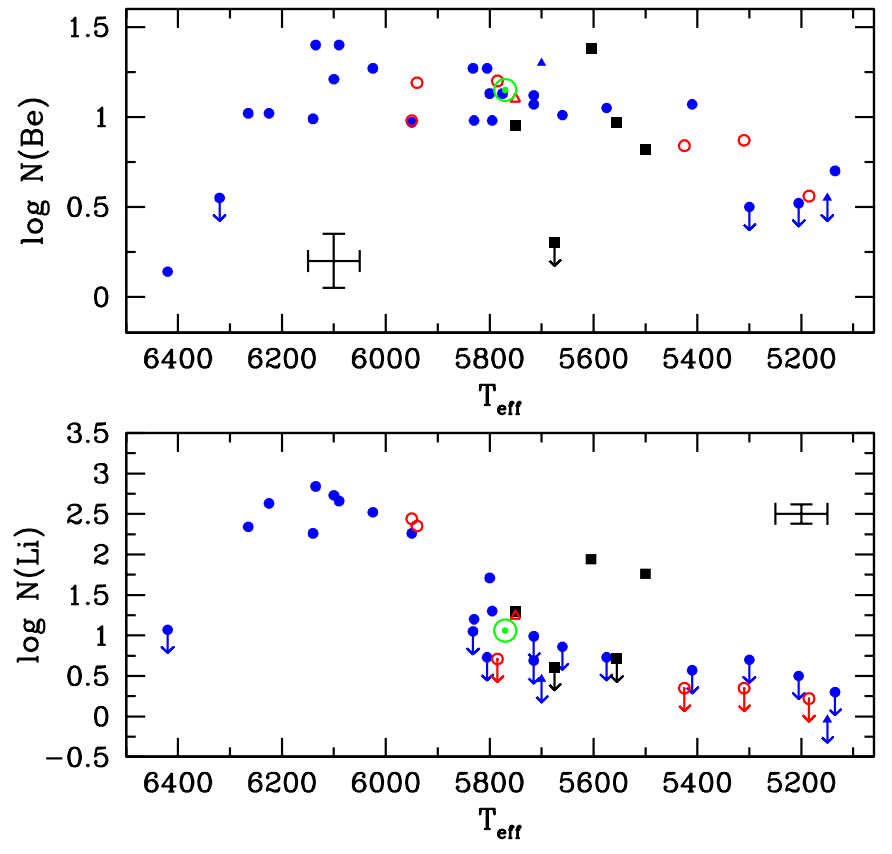

Fig. 4. Lithium and Beryllium abundances for the stars studied in this paper. We note that for some of the stars, no Li abundances were found in the literature (see Table 2). Symbols as in Fig. 3. Typical error bars are represented in each panel (0.16 for Be and 0.12 for $\mathrm{Li}$ ).

case for $14 \mathrm{Her}$, with an age around $\sim 6$ Gyr. Furthermore, for 14 Her, there is a star (HD 222335, without detected giant planet) with about the same temperature, similar age ( $\sim 5-6 \mathrm{Gyr})$ but much higher Be abundance.

Can a metallicity effect explain the observed difference? An increase in the metallicity (and thus in the opacities) has the effect of changing the convective envelope depth of a star. It has been shown by Swenson et al. (1994) that even small changes in the oxygen abundances may have a strong effect on the Li depletion rates. Although it is unwise to extrapolate directly, a similar effect should exist concerning Be. In what concerns our case, most of the objects in the plot having temperatures lower than $5400 \mathrm{~K}$ are metal-rich with respect to the Sun. For example, $14 \mathrm{Her}$ is one of the most metal rich stars in the sample $([\mathrm{Fe} / \mathrm{H}]=+0.50)$. But some counter examples exist, like Gl 86 , only about $100 \mathrm{~K}$ cooler, with a value of $[\mathrm{Fe} / \mathrm{H}]=-0.20$. Unfortunately, no theoretical isochrones for Be depletion are available for these high metallicities, and it is not clear whether a difference of +0.5 dex in metallicity can or cannot induce significantly different $\mathrm{Be}$ depletion rates for this temperature range. On the other hand, if some unknown opacity effect could lead to systematic errors as a function of temperature e.g. the missing UV opacity (Balachandran \& Bell 1998) we could maybe expect to measure some trend. However, we have no special reason to believe that this effect can lead to the observed trend. Furthermore, the missing UV opacity problem is far from being solved (if it exists at all); indeed, recent results seem to show that we can obtain a 
good fit of the solar spectrum without taking into account this extra-opacity (Allende Prieto \& Lambert 2000).

Although not conclusive, the evidences discussed above suggest that a bona-fide explanation for the observed Be abundances may pass either by some inconsistency in the models, or by some metallicity effect. But given the much higher number of planet hosts in the plot when compared with the non-planet hosts, we still cannot discard that the presence of a planet might be the responsible for the observed trend ${ }^{9}$. Else, since Be depletion in the Pinsonneault et al. (1990) models is strongly related to the angular momentum lost, we would have, for example, to consider that there is some negative correlation between initial angular momentum and/or angular momentum loss and stellar mass (which does not seem very plausible). Unfortunately only the addition of more objects to the plot, and in particular the determination of $\mathrm{Be}$ abundances for a larger sample of dwarfs with $T_{\text {eff }}<5400 \mathrm{~K}$ will permit to better settle down this question. Such observations are currently in progress.

It is also worth mentioning that on the other side of the $T_{\text {eff }}$ plane, both HD 120136 ( $\tau$ Boo) and BD - 103166, positioned in the $\mathrm{Li}$ (and Be) dip region (e.g. Boesgaard \& King 2002), have a Be abundance that may be compatible with the models.

\subsection{Lithium vs. beryllium}

Our data can also be used to further investigate the issue of $\mathrm{Li}$ and Be depletion in main sequence stars. Figure 4 compares $\mathrm{Li}$ and $\mathrm{Be}$ abundances for the same stars, and shows, as expected, that $\mathrm{Li}$ is burned much faster than $\mathrm{Be}$, and its decline with temperature is much more clear. This is clearly expected from the models for a middle age solar type dwarf in this temperature regime. Only two points seem to come out of the main trend (in the $\mathrm{Li}$ panel). These are the cases of HD 10697 and HD 117176 (70 Vir). These two stars deserve some particular discussion in Sect. 4.2.1.

In Fig. 5 we plot Be vs. Li abundances for all the stars with $T_{\text {eff }}<6300 \mathrm{~K}$ for which both $\mathrm{Li}$ and Be abundances are available. The plot reveals no clear trends. Even 70 Vir and HD 10697 follow the main trend, probably attesting the normality of these two stars. Again, no significant difference is seen between the two groups of stars (planet hosts and non-planet hosts).

One interesting but expected detail in Fig. 5 is that there are no stars in the lower right part of the plot, a region that would correspond to stars having depleted much of their Be but that are still Li rich. This "gap" in the figure, plus the few stars in the upper right corner, suggests that there might be a correlation between $\mathrm{Li}$ and $\mathrm{Be}$ depletions (like the one found by Deliyannis et al. 1998;

\footnotetext{
${ }^{9}$ Can the presence of a massive proto-planetary disk (able to form planets), or other variables connected to the process of planetary formation itself (e.g. timescale), induce different angular momentum histories for stars with different $T_{\text {eff }}$ ?
}

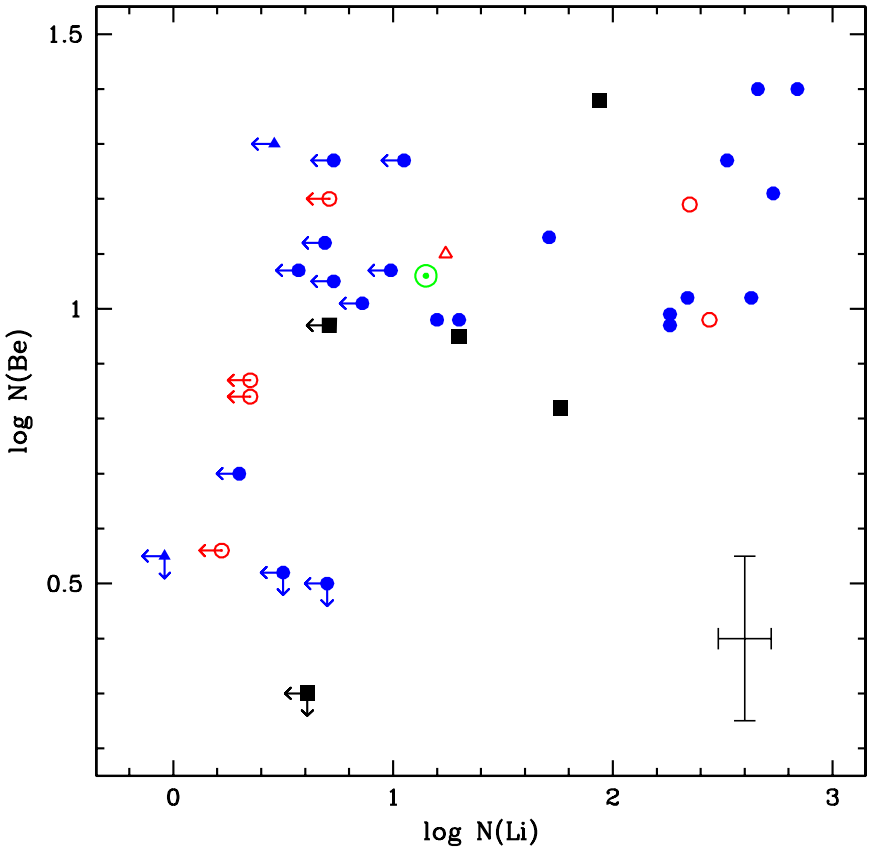

Fig. 5. Li vs. Be abundances for the stars in our sample having temperatures lower than $6300 \mathrm{~K}$ and for which both $\mathrm{Li}$ and Be abundances were available. Symbols as in Fig. 3. Typical error bars are represented in the lower-right corner of the figure.

Boesgaard et al. 2001 and more recently by Boesgaard \& King 2002 for their sample of hotter dwarfs, but absent in the study of Domínguez Herrera 1998, and in the sample of old cluster members of Randich et al. 2002). However, there is a significant number of stars in the upper left region of the plot having Be determinations but only upper limits for the Li abundance (objects that are not present in Fig. 14 of Boesgaard et al.). This particular point in quite interesting, since it is, to our knowledge, the first time such a "population" of stars is discussed in the literature.

In Fig. 6 we have done the same plot as in Fig. 5, but this time using different symbols as a function of the temperature of the object. As we can easily see from the plot, the objects in the three different temperature regimes chosen occupy clearly different positions in the diagramme. Stars with $T_{\text {eff }} \leq 5400$ are positioned in the lower-left corner. These correspond basically to objects having burned some Be, and that only have upper values for the Li abundance. In the upper-right corner of the figure are positioned stars in the range $T_{\text {eff }}>5900 \mathrm{~K}$. These correspond to objects that have essentially not burned any Be, having depleted only moderately their Li content. An intermediate population, with $5400 \mathrm{~K}<T_{\text {eff }} \leq 5900 \mathrm{~K}$, having already considerably depleted its original $\mathrm{Li}$ but not its $\mathrm{Be}$ content is present in the mid-upper part of the plot.

Except for HD 38529, a sub-giant star (the object with the lowest Be abundance in the plot), all the other objects are well grouped according to their temperature range. This is an interesting result, since it is giving us important clues about the temperature at which the onset of $\mathrm{Li}$ and Be depletion occurs in our metal-rich stars: if for $\mathrm{Li}$ 


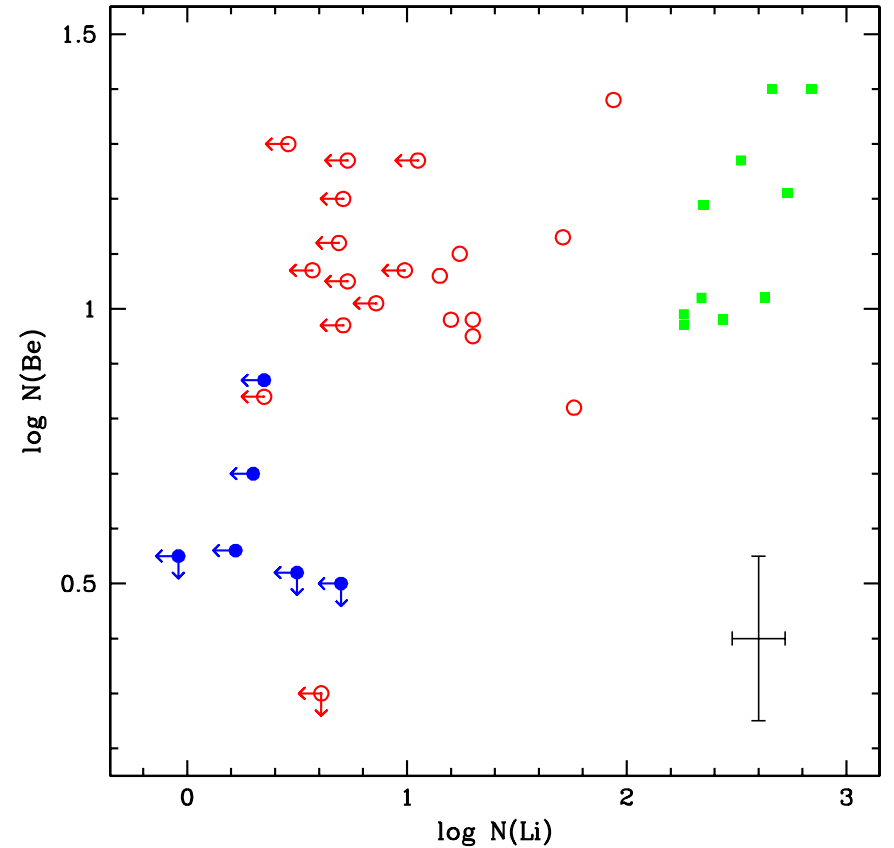

Fig. 6. Same as Fig. 5 but using different symbols for three temperature intervals: $T_{\text {eff }} \leq 5400 \mathrm{~K}$ (filled circles), $5400 \mathrm{~K}<$ $T_{\text {eff }} \leq 5900 \mathrm{~K}$ (open circles), and $T_{\text {eff }}>5900 \mathrm{~K}$ (filled squares). See text for more details.

depletion occurs already for temperatures around $5900 \mathrm{~K}$, for Be this only seems to happen at $T_{\text {eff }} \sim 5400 \mathrm{~K}$. Note, however, that given the dispertion these limits have to be seen as approximate.

Note also that we did not include $\tau$ Boo in Figs. 5 and 6 , since its the only object in the sample that is clearly in the Li-dip region.

\subsubsection{HD 10697 and 70 Vir}

As mentioned above, HD 10697 and 70 Vir do not fit into the mean trend in the lower panel of Fig. 4. Considering as initial abundances of $\mathrm{Li}$ the "cosmic" value $(\log N(\mathrm{Li})=$ 3.31; Martin et al. 1994) and for Be the average between meteoritic and solar $(\log N(\mathrm{Be})=1.28$, as above), then the observed values for $70 \mathrm{Vir}$ indicate that $\mathrm{Be}$ $(\log N(\mathrm{Be})=0.82)$ is depleted about 3 times, while Li $(\log N(\mathrm{Li})=1.76)$ is depleted by by a factor of 22 . Nevertheless, such a pattern of Li and Be depletion is not so unexpected (see e.g. Deliyannis \& Pinsonneault 1997; Stephens et al. 1997), and is compatible with models of depletion including turbulent induced rotational mixing (see e.g. discussion in Stephens et al. 1997). But 70 Vir is not a young object (it seems to be slightly evolved, as confirmed by its low surface gravity $\log g=3.90$ ), and it seems strange that an evolved star with its temperature may still have so much Li.

One possibility would be to invoke Li and Be dredge up from a "buffer" below the former main-sequence convective envelope (Deliyannis et al. 1990). But as discussed in e.g. Randich et al. (1999), this scenario does not seem to be supported by the observations. However, the fact that this star is evolved means that its current temperature is probably different to the one it had during the main-sequence phase. With a near solar metallicity $([\mathrm{Fe} / \mathrm{H}]=-0.03)$ and a mass of $\sim 1.1 M_{\odot}$, the temperature of $70 \mathrm{Vir}$ would have been more close to $6000 \mathrm{~K}$. A dwarf with this temperature is not supposed to burn much of its $\mathrm{Li}$ (as we can see from the plot). So, we speculate that this star may have just left the main-sequence, and started to dilute (and/or burn) the $\mathrm{Li}$ (as well as Be) content present in its convective envelope. If the size of the convective envelope has increased "quite fast", maybe it still did not have time to deplete/dilute all the $\mathrm{Li}$, but is already depleting part of its Be (Charbonnel et al. 2000).

The case of HD 10697 is in fact not very different from the one of $70 \mathrm{Vir}$. Both have the same mass $\left(1.10 M_{\odot}\right)$, and similar surface gravities. The main difference resides in the fact that HD 10647 seems to have, within the errors, preserved most of its Be content, while $\mathrm{Li}$ is depleted about 23 times. But given the uncertainties in our Be abundance determination, and the unknown initial $\mathrm{Li}$ and Be content of the star, we cannot be certain whether the Be is really intact in the atmosphere of this object. The same arguments discussed for 70 Vir may thus be valid in this case.

It is worth mentioning that another possibility to explain the high Li abundances of these two stars would be to invoke a planet engulfment (like in the case of HD 82943 - Israelian et al. 2001a), that could simply result from the migration of a former close-in planet due to tidal interactions with the evolved star (Rasio et al. 1996). Unfortunately, no measurements of the $\mathrm{Li}$ isotopic ratio are available for 70 Vir. But recent models by Siess \& Livio (1999) show that the absorption of a planet by a giant star would have the effect of increasing considerably its Li abundance, but only slightly the Be content, a situation that could be compatible with the observed $\mathrm{Li}$ and Be abundances for this star.

There is another interesting point about these two stars. Although they have very similar Li abundances, their Be contents differ by 0.56 dex. Given that both stars have similar metallicity $(-0.03$ and +0.16 , for $70 \mathrm{Vir}$ and HD 10697, respectively) their initial Be content is not expected to be significantly different (Rebolo et al. 1988; Molaro et al. 1997; García López et al., in preparation). However, we do not know up to which extent Be and $\mathrm{Li}$ abundances are uniform in the Inter-Stellar Medium. A different initial $\mathrm{Li} / \mathrm{Be}$ ratio, together with the uncertainties in the $\mathrm{Li}$ and Be determinations, could probably lead to the observed effect. Else, we would need to invoke that the two stars have burned $\mathrm{Li}$ at the same rate, while the opposite happened for Be. But given that these two stars are sub-giants, we cannot exclude that we are simply observing some evolutionary effect, eventually combined with slightly different initial $\mathrm{Li}$ and Be abundances. Note that the former object is a bit cooler, being positioned in the temperature region where the Be depletion seems to occur (see previous section). 


\section{Conclusions}

We have obtained Be abundances for a sample of planet hosts stars, and a smaller sample of stars without known giant planetary companions, aimed at studying the existence of any significant difference between the two groups. Our data also allow us to further investigate Li and Be depletion among metal-rich stars. The objects have metallicities between about -0.6 and $+0.5 \mathrm{dex}$, and cover the temperature interval between $\sim 5150 \mathrm{~K}$ and $6450 \mathrm{~K}$. The main conclusions go as follows:

- No particular difference was found between the Be abundances of stars with and without giant planetary companions in the temperature interval for which both groups were observed. This may be interpreted as an argument against the "pollution scenario" as the source of the high- $[\mathrm{Fe} / \mathrm{H}]$ observed for planet host stars. However, given the low number of comparison stars available in this study, no strong constraints can still be set.

- For stars with $T_{\text {eff }}<5500 \mathrm{~K}$, the general trend of the Be abundances is not well described by models of light element depletion for solar metallicity (e.g. Pinsonneault et al. 1990). These inconsistencies may be connected either to some metallicity effect, or to some problem with the models, but at the moment we cannot completely discard some relation with the presence of a planet.

- For temperatures lower than $6300 \mathrm{~K}$, no clear correlated $\mathrm{Li}$ and Be pattern is seen, contrary to what was found by other authors for hotter dwarfs (Deliyannis et al. 1998; Boesgaard et al. 2001).

- Our data permit to establish approximate temperature limits for the onset of strong $\mathrm{Li}$ and Be depletion for the metal-rich stars studied in this work. For stars with $T_{\text {eff }}<5900 \mathrm{~K}$, severe Li burning is seen, while for Be this limit is around $5400 \mathrm{~K}$.

A large survey for Be in planet and non-planet host stars is currently under way, and we expect it to give new insights into this timely and interesting subject.

Acknowledgements. We thank our anonymous referee for the useful and interesting comments and suggestions. We wish to thank the Swiss National Science Foundation (FNSRS) for the continuous support to this project. This research was also partially supported by the Spanish DGES under project PB98-0531-C02-02. Support from Fundação para a Ciência e Tecnologia, Portugal, to N.C.S. in the form of a scholarship is gratefully acknowledged.

\section{References}

Allende Prieto, C., \& Lambert, D. L. 2000, AJ, 119, 2445

Anders, R., \& Grevesse, N. 1989, Geochim. Cosmochim. Acta, 53, 197

Balachandran, S. C., \& Bell, R. A. 1998, Nature, 392, 791

Barnes, S., Sofia, S., \& Pinsonneault, M. 2001, ApJ, 548, 1071

Boesgaard, A. M., \& King, J. R. 2002, ApJ, 565, 587
Boesgaard, A. M., Deliyannis, C. P., King, J. R., \& Stephens, A. 2001, ApJ, 553, 754

Benz, W., \& Mayor, M. 1984, A\&A, 138, 183

Charbonnel, C., Deliyannis, C. P., \& Pinsonneault, M. 2000, in The light Elements and their Evolution, ed. L. da Silva, M. Spite, \& J. R. de Medeiros, IAU Symp. 198, ASP Conf. Ser., 198, 87

Chmielewski, Y., Müller, E. A., \& Brault, J. W. 1975, A\&A, 42,37

Cochran, W. D., Hatzes, A. P., Butler, R. P., \& Marcy, G. W. 1997, ApJ, 483, 457

Deliyannis, C. P., Cunha, K., King, J. R., \& Boesgaard, A. M. 2000, AJ, 119, 2437

Deliyannis, C. P., Boesgaard, A. M., Stephens, A., et al. 1998, ApJ, 498, L147

Deliyannis, C. P., \& Pinsonneault, M. H. 1997, ApJ, 488, 836

Deliyannis, C. P., \& Pinsonneault, M. H. 1993, in IAU Colloq. 137, Inside the Stars, ed. W. W. Weiss, ASP Conf. Ser., 40, 174

Deliyannis, C. P., Demarque, P., \& Kalawer, S. 1990, ApJS, 73,21

Domínguez Herrera, M. C. 1998, Masters Thesis, University of La Laguna, Spain

Edwards, S., Strom, S. E., Hartigan, P., et al. 1993, AJ, 106, 372

García López, R., Santos, N. C., Israelian, G., et al. 2001, in proceedings of the 12th workshop on Cool Stars, Stellar Systems, and the Sun

García López, R., \& Perez de Taoro, M. R. 1998, A\&A, 334, 599

García López, R., Severino, G., \& Gomez, M. T. 1995, A\&A, 297, 787

García López, R. J., Rebolo, R., \& Martín, E. L. 1994, A\&A, 282,518

Gonzalez, G., Laws, C., Tyagi, S., \& Reddy, B. E. 2001, AJ, 121,432

Gonzalez, G., \& Laws, C. 2000, AJ, 119, 390

Gonzalez, G., Wallerstein, G., \& Saar, S. 1999, ApJ, 511, L111

Gonzalez, G. 1998, A\&A, 334, 221

Hartmann, L. 2002, ApJ, 566, L29

Israelian, G., Santos, N. C., Mayor, M., \& Rebolo, R. 2001a, Nature, 411, 163

Israelian, G., Santos, N. C., Mayor, M., \& Rebolo, R. 2001b, $\mathrm{A} \& \mathrm{~A}$, in preparation

Jones, B. F., Fisher, D., \& Soderblom, D. R. 1999, AJ, 117, 330

King, J. R., Deliyannis, C. P., Hiltgen, D. D., et al. 1997, AJ, 113,1871

Kurucz, R. L. 1993, CD-ROMs, ATLAS9 Stellar Atmospheres Programs and $2 \mathrm{~km} \mathrm{~s}^{-1}$ Grid (Cambridge: Smithsonian Astrophys. Obs.)

Laughlin, G. 2000, ApJ, 545, 1064

Laws, C., \& Gonzalez, G. 2001, ApJ, 553, 405

Marcy, G. W., Cochran, W. D., \& Mayor, M. 1999, in Protostars, \& Planets IV, ed. V. Mannings, A. Boss, \& S. Russel (University of Arizona Press, Tucson)

Martin, E. L., Rebolo, R., Magazzù, A., \& Pavlenko, Y. V. 1994, A\&A, 282, 503

Mayor, M., \& Santos, N. C. 2001, in The Origin of Stars and Planets: the VLT View, ed. J. Alves, \& M. McCaughrean, in press

Mayor, M., \& Queloz, D. 1995, Nature, 378, 355

Molaro, P., Bonifacio, P., Castelli, F., \& Pasquini, L. 1997, A\&A, 319, 593 
Pinsonneault, M. H., DePoy, D. L., \& Coffee, M. 2001, ApJ, $556, \mathrm{~L} 59$

Pinsonneault, M. H., Kawaler, S. D., \& Demarque, P. 1990, ApJS, 74, 501

Pollack, J. B., Hubickyj, O., Bodenheimer, P., et al. 1996, Icarus, 124,62

Randich, S., Primas, F., Pasquini, L., \& Pallavicini, R. 2002, $\mathrm{A} \& \mathrm{~A}$, in press

Randich, S., Gratton, R., Pallavicini, R., Pasquini, L., \& Carretta, E. 1999, A\&A, 348, 487

Randich, S., Martín, E. L., García López, R., \& Pallavicini, R. 1998, A\&A, 333, 591

Rasio, F. A., Tout, C. A., Lubow, S. H., \& Livio, M. 1996, ApJ, 470, 1187

Rebolo, R., Molaro, P., Abia, C., \& Beckman, J. E. 1988, A\&A, 193, 193

Rebull, L. M. 2001, AJ, 121, 1676

Reeves, H. 1994, Rev. Mod. Phys., 66, 193

Santos, N. C., Israelian, G., \& Mayor, M. 2001a, A\&A, 373, 1019

Santos, N. C., Israelian, G., \& Mayor, M. 2001b, in Proc. of the 12th workshop on Cool Stars, Stellar Systems, and the Sun

Santos, N. C., Israelian, G., \& Mayor, M. 2000, A\&A, 363, 228
Schaerer, D., Meynet, G., Maeder, A., \& Schaller, G. 1993a, A\&AS, 98, 523

Schaerer, D., Charbonnel, C., Meynet, G., Maeder, A., \& Schaller, G. 1993b, A\&AS, 102, 339

Schaller, G., Schaerer, D., Meynet, G., \& Maeder, A. 1992, A\&AS, 96, 269

Siess, L., \& Livio, M. 1999, MNRAS, 304, 925

Sneden, C. 1973, Ph.D. Thesis, University of Texas

Stassun, K. G., Mathieu, R. D., Mazeh, T., \& Vrba, F. J. 1999, AJ, 117, 2941

Stephens, A., Boesgaard, A. M., King, J. R., \& Deliyannis, C. P. 1997, ApJ, 491, 339

Strom, S. E. 1994, in 8th workshop on Cool Stars, Stellar Systems, and the Sun, ed. J.-P. Caillault, ASP Conf. Ser., 64, 211

Swenson, F. J., Faulkner, J., Iglesias, C. A., Rogers, F. J., \& Alexander, D. R. 1994, ApJ, 422, L79

Udry, S., Mayor, M., Naef, D., et al. 2000, A\&A, 356, 590

Udry, S., Mayor, M., \& Queloz, D. 2001, in Planetary Systems in the Universe: Observations, Formation and Evolution, ed. A. Penny, P. Artimowicz, A.-M. Lagrange, \& S. Russel, ASP Conf. Ser., in press 